KOMPASS

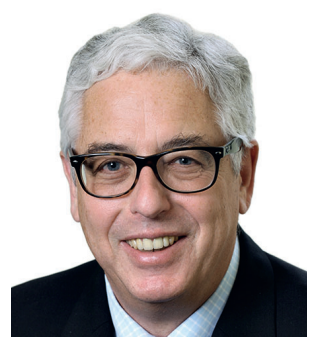

Percy Lehmann

HELIOS Universitätsklinikum Wuppertal, Zentrum für Dermatologie, Allergologie, Dermatochirurgie, DKG zertifiziertes

Hauttumorzentrum, Universität WittenHerdecke, Deutschland

Die photodynamische Therapie (PDT) beschreibt eine photochemische Reaktion, die nach Absorption von Licht durch einen Photosensibilisator reaktive Sauerstoffspezies generiert. Dadurch können Tumorzellen zerstört und Entzündungskaskaden modifiziert werden [1].

Die ersten Arbeiten zur photodynamischen Therapie stammen aus der Arbeitsgruppe von Tappeiner, der auch den Terminus «photodynamisch» aufgrund seiner experimentellen Arbeiten 1904 prägte [2]. Schon am Anfang wurden Patienten mit Basalzellkarzinomen und infektiösen Erkrankungen wie dem Lupus vulgaris zusammen mit dem Dermatologen Albert Jesionek behandelt und beschrieben [3]. In den folgenden 100 Jahren erlebte diese Therapieform eine wechselhafte Geschichte bis zur klinischen Zulassung.

Seit Anfang dieses Jahrhunderts hat die PDT einen festen Platz in der Therapiestrategie von Dermatologen und erfreut sich zunehmender Beliebtheit. Bei der Behandlung von zahlreichen aktinischen Keratosen in größeren Regionen (Feldkanzerisierung) gilt sie für viele Experten als Methode der 1. Wahl. Zahlreiche Studien, die die therapeutische Effektivität, das kosmetische Ergebnis, Patientenpräferenz sowie die Nebenwirkungsrate beinhalten, führten zu dieser herausragenden Stellung. Als weitere gesi-

\title{
Die photodynamische Therapie entwickelt sich weiter
}

cherte Indikationen mit hoher Präferenz gelten neben der Feldkanzerisierung mit aktinischen Keratosen das superfizielle Basalzellkarzinom und der Morbus Bowen [4]. Neben diesen UV-induzierten epithelialen Tumoren, die für die häufigsten Krebsarten des Menschen verantwortlich sind und in den letzten Jahrzehnten die größte Inzidenzzunahme aller Krebsarten aufweisen, spielt eine weitere zunehmende Population eine große Rolle in unserem klinischen Alltag. Dies sind organtransplantierte Patienten, die durch ihre Immunsuppression ebenso von epithelialen Hautkrebsarten stark betroffen sind.

In Deutschland leben zurzeit etwa 80000 bis 90000 transplantierte Patienten, die einer engmaschigen dermatologischen Kontrolle bedürfen. Durch die Verlängerung der Überlebenszeit stieg die Inzidenz von Hauttumoren enorm an. Innerhalb der ersten 5 Jahre treten bei $40 \%$ der Patienten maligne und prämaligne Hautveränderungen auf. Auch für diese Patienten hat sich die PDT als sehr geeignet erwiesen. Es konnte sogar nachgewiesen werden, dass durch eine zyklische photodynamische Therapie z.B. alle 3 oder 6 Monate das Neuauftreten von prämalignen und malignen epithelialen Hautkrebsarten verhindert werden kann.

Als wesentlicher Nachteil bei der Behandlung größerer Flächen mit konventioneller
PDT erweist sich nach wie vor die deutliche Schmerzhaftigkeit während der Bestrahlung, so dass einige Patienten diese Prozedur nicht wiederholen möchten. Daher erfuhr die erste Publikation zur TageslichtPDT, die eine fast schmerzlose Bestrahlung bei guter Effektivität beschrieb, großes Interesse. Es zeigte sich, dass das kontinuierliche Anfluten des Protoporphyrin IX in die Zielzelle bei gleichzeitiger Bestrahlung zur signifikanten Reduktion der Schmerzhaftigkeit führte [5]. Um diese Therapie besser kontrollierbar zu machen, wurden konsekutiv verschiedene Strahlenquellen entwickelt, die dem Sonnenlicht entsprechen und so auch eine klimaunabhängige PDT mit Tageslicht ermöglichten [6, 7]. Weitere Studien müssen jedoch folgen, um mit gut konzipierten Bestrahlungsquellen sowie definierten physikalischen Parametern und Dosimetrie bei den verschiedenen Indikationen den Weg in die Zukunft weiter beschreiten zu können.

Die wesentlichen Vorteile und Verbesserungen konventioneller phototherapeutischer Strategien wurden in den letzten Jahrhunderten durch die pharmakologische Entwicklung von wirksamen Medikamenten (z.B. Psoralene) und durch die Entwicklung leistungsstarker Strahlenquellen mit definierten Wellenlängen und Dosimetrie erreicht. Hierdurch konnte man für ver-

\section{KARGER}

Fax +497614520714

information@karger.com

www.karger.com
(๑) 2019 S. Karger GmbH, Freiburg

Accessible online at:

www.karger.com/kkd
Prof. Dr. Percy Lehmann

HELIOS Universitätsklinikum Wuppertal, Universität Witten Herdecke

Dermatologie - Hautklinik

Heusnerstraße 40, Haus 5 und 4, 42283 Wuppertal, Deutschland

Percy.Lehmann@helios-gesundheit.de 
schiedene Dermatosen die optimalen Bestrahlungsmodalitäten erforschen mit spezifischen Bestrahlungsprotokollen. Als Meilensteine der Entwicklungen können hierbei die PUVA-Therapie (Psoralene + UVB), die selektive UV-B-Bestrahlung und deren Weiterentwicklung, die Schmalspektrum-UV-B-Bestrahlung (Bereich 311 bis 313 $\mathrm{nm}$ ), in Kombination mit verschiedenen Externa wie Vitamin D3-Analoga genannt werden [8].

Dem Beispiel der konventionellen Photo- therapie folgend, werden auch für die PDT durch immer neue Studien die optimalen Strahlenquellen und Photosensibilisatoren sowie die entsprechende Dosimetrie erforscht. In diesem Sinne kommentiert Dr. Philipp-Dormston in diesem Heft eine Arbeit von Maytin EV et al, die die Effektivität von blauem Licht gegenüber rotem Licht in der Therapie von Basalzellkarzinomen bei Patienten mit Gorlin-Goltz-Syndrom überprüft. Dies ist eine bilateral kontrollierte Vergleichsstudie und es ist zu hoffen, dass durch die weitere Erforschung mit entsprechender Methodik die Basis für eine optimale Patientenversorgung im klinischen Alltag geschaffen wird.

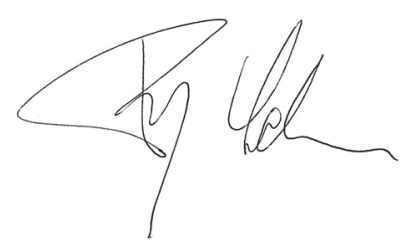

Percy Lehmann

\section{Literatur}

1 Fritsch C, Lehmann P, Stahl W, Schulte KW, Blohm E, Lang K, Slies H, Ruzicka T: Optimum porphyrin accumulation in epithelial skin tumours and psoriatic lesions after topical application of $\delta$-aminolaevunic acid. Br J Cancer 1999;79:1603-1608.

2 Von Tappeiner H: Die photodynamische Erscheinung. Ergebn Physiol 1909;8:698-741.

3 Von Tappeiner H, Hesionek A: Therapeutische Versuche mit fluoreszierenden Stoffen. Münch Med Wochenschr 1903;50:2042-2044.
4 Braathen LR, Szeimies RM, Basset-Seguin N, Bissonette R, Foley P, Pariser D, Roelandts R, Wennberg AM, Mortin CA: Guidelines on the use of photodynamic therapy for nonmelanoma skin cancer: an international consensus. J Am Acad Dermatol 2007;56:125-143.

5 Lehmann P (2017) Tageslicht-photodynamische Therapie: Zurück in die Zukunft? Hautarzt 2017;69(2):180-183.

6 Lerche CM, Heerfordt IM, Heydenreich J, Wulf HC: Alternatives to outdoor daylight illumina- tion for photodynamic therapy - use of greenhouses and artificial light sources. Int J Mol Sci 2016;17:309.

7 Philipp-Dormston WG, Karrer S, Petering H, Ulrich C, Dirschka T, Berking C, Lonsdorf AS, Gerber PA, Radkovic S, Hunger RE, Szeimies RM: Daylight PDT with MAL - current data and practical recommendations of an expert panel. J Dtsch Dermatol Ges 2015;13:1240-1249.

8 Hönigsmann H: Phototherapy. J Invest Dermatol 2013;133:18-20. 